\title{
Modeling the permittivity of two-phase media containing monodisperse spheres: Effects of microstructure and multiple scattering
}

\author{
Timothy E. Doyle \\ Department of Physics, Utah State University, Logan, Utah 84322-4415, USA \\ David A. Robinson \\ Department of Geophysics, Stanford University, Stanford, California 94305-2215, USA \\ Scott B. Jones \\ Department of Plants, Soils, and Climate, Utah State University, Logan, Utah 84322-4820, USA \\ Keith H. Warnick and Brent L. Carruth \\ Department of Physics, Utah State University, Logan, Utah 84322-4415, USA
}

(Received 30 March 2007; published 7 August 2007)

\begin{abstract}
A numerical modeling approach was developed to predict the dielectric properties of heterogeneous particulate materials with arbitrary microstructures. To test the method, simulation and experimental data were acquired for the effective permittivities of various glass sphere suspensions. Both ordered lattices and random microstructures of up to 3600 spheres were modeled for volume fractions of $0.025-0.60$. The electric fields in the suspensions were computed using an iterative multipole method that included multiple-scattering effects. The effective permittivities were obtained by averaging the electric field and electric displacement over a representative volume. Frequency spectra, electric field images, and single-scattering results (i.e., no particleparticle interactions) were additionally generated. The results were compared to experimental data for random close-packed microstructures, to effective-medium approximations, to exact lattice models, and to a perturbation expansion model. The comparisons showed that the iterative models agreed with the exact lattice models to within $3.31 \%$ for crystalline suspensions. Results for random suspensions agreed with the perturbation expansion model to within $1.76 \%$ for volume fractions up to 0.50 . Single-scattering models additionally predicted permittivities for the microstructures as well as or better than the Maxwell Garnett approximation [Philos. Trans. R. Soc. London, Ser. A 203, 385 (1904)], suggesting that microstructural effects and multipole moments higher than the dipole are required for more accurate statistical prediction of effective permittivities. The effective permittivities, convergence behavior, and dispersion behavior of the simulations were sensitive to both microstructure and the extent of multiple scattering included in the models, illustrating how the macroscopic properties depend significantly on the microscopic details of the interactions. In contrast to other approaches, the iterative multipole method can model both the frequency and spatial dependencies of the electromagnetic properties of particulate materials, as well as a wide variety of microstructures, including polydisperse and hierarchical systems.
\end{abstract}

DOI: 10.1103/PhysRevB.76.054203

PACS number(s): 77.84.Lf, 82.70.Kj, 42.25.Dd

\section{INTRODUCTION}

Determining the effective electromagnetic properties of heterogeneous mixtures has been a continuing interest in physics and other scientific fields for over 150 years since the initial work of Faraday and Maxwell. ${ }^{1,2}$ A suspension of spherical particles in a homogeneous matrix is the archetypal example that has been investigated extensively. Although much success has been achieved with mean-field theory and other statistical approximations, the effects of local structure (microstructure) on the macroscopic properties remain an intriguing and important problem. Predicting the effects of various particle configurations, mixed particle compositions, and multimodal size distributions continues to be a challenge for predicting the dielectric properties of soils, composites, biomaterials, and other random or complexly structured media. Mapping the spatial distribution of the electromagnetic field intensity at the microscopic level is also vital for understanding phenomena such as dielectric breakdown in com- posites or $\mathrm{rf}$ and/or microwave exposure in living tissues.

The Clausius-Mossotti formula (also known as the Lorenz-Lorentz formula) is a first-order approximation for the dielectric properties of mixtures, but contains parameters more relevant to describing molecular effects (e.g., in dilute gases) than heterogeneous mixtures with inclusions. ${ }^{3}$ In 1904, Maxwell Garnett rederived a macroscopic version of the Clausius-Mossotti formula to describe the effective refractive index and/or dielectric properties of a suspension of spheres, and the Maxwell Garnett formula is still commonly used for two-phase mixtures to this day. ${ }^{4}$ More recent and sophisticated effective-medium, self-consistent, and other approximation methods have been developed. Torquato provides an extensive review of these methods. ${ }^{5}$ Of particular note are the self-similar or differential effective-medium approach $^{6}$ and a generalized approach that combines different approximations into a single formula. ${ }^{7}$ Approximation methods have also been extended to include multiphase, anisotropic, and chiral mixtures as well as mixtures containing 
ellipsoidal and layered particles. ${ }^{3,5}$ Most of these formulas remain statistical in nature, however, deriving from averaged field interactions rather than a first principles description of all multiple interactions between particles. One exception is the perturbation expansion method developed by Torquato that includes microstructural input via three-point probability functions. ${ }^{8}$

Multipole expansion methods are efficient and powerful tools for modeling suspensions of spherical particles due to the separability of the Laplace and Helmholtz equations in spherical coordinates. The additional symmetry provided by ordering the particles into periodic microstructures has allowed the conductivity to be solved for simple-cubic (sc), body-centered-cubic (bcc), and face-centered-cubic (fcc) lattices. $^{9-12}$ These models are generally based on the Rayleigh method, and similar methods have been applied to lattices of spheroids. ${ }^{13-15}$ Other approaches are required, however, for modeling electrostatic and electromagnetic fields in arbitrary configurations of spheres. These approaches include iterative, ${ }^{16-21} T$-matrix, ${ }^{22-26}$ fast multipole, ${ }^{27-30}$ and other matrix methods. ${ }^{31}$ Inherent to all of these multipole approaches is the determination of effective properties by directly computing the microscopic interactions. This is achieved by calculating the multiple-scattering effects with the use of addition theorems, translation operators, or multipole interaction tensors.

Iterative multipole (IM) methods provide a general approach for simulating the dielectric properties of suspensions with fewer constraints on the microstructure than other methods (e.g., the suspension does not have to be ordered, isotropic, or homogeneous on a macroscopic scale). The approach can, therefore, be tested against exact lattice methods to verify its accuracy and can be applied to random or hierarchically structured media. Clusters of inclusions of finite size can also be modeled with the iterative multipole method. Such systems may have different dielectric properties than an infinite lattice or random microstructure, a phenomenon analogous to the size effect observed for the dielectric properties of some nanoparticles (where the inclusions would correspond to the atoms in the nanoparticle's crystal lattice). Finally, a direct iterative approach is computationally simpler and more tractable than other approaches.

In this work, an iterative multipole approach was developed to simulate electromagnetic fields in suspensions containing up to 3600 spherical particles. The approach used vector multipole expansions, partial wave expansions, Mie scattering solutions, and addition theorems to compute the multiple-scattering interactions and resultant fields. The inclusions in the simulated media were configured into four different lattice types and two different random arrangements. The effective permittivities of the suspensions were determined for particle-volume fractions up to 0.60. The simulation results were compared to experimental data from close-packed glass beads, results from effective-medium approximations, and predictions from exact lattice calculations. The accuracy, convergence behavior, and stability of the model were also determined as a function of the highest computed multipole order, iteration step, and number of particles in the simulation. Finally, the dielectric frequency spectrum and spatial distribution of electromagnetic field in- tensities were simulated for a fcc lattice and random suspension.

\section{METHODS}

\section{A. Multipole theory}

The iterative multipole approach models the electromagnetic fields of a spherical particle or inclusion with vector multipole functions derived from the Maxwell equations. The Maxwell equations for a macroscopic dielectric medium with no free charges can be reformulated as a pair of wave equations:

$$
\begin{aligned}
& \nabla^{2} \mathbf{H}-\frac{\varepsilon \mu}{c^{2}} \frac{\partial^{2} \mathbf{H}}{\partial t^{2}}=0, \\
& \nabla^{2} \mathbf{E}-\frac{\varepsilon \mu}{c^{2}} \frac{\partial^{2} \mathbf{E}}{\partial t^{2}}=0 .
\end{aligned}
$$

$\mathbf{E}$ and $\mathbf{H}$ are the electric and magnetic fields, respectively, and $\varepsilon, \mu$, and $c$ are the electric permittivity, magnetic permeability, and speed of light. Assuming a purely sinusoidal, steady-state time dependence for the electric and magnetic fields of the form $\mathbf{E}(\mathbf{x}, t)=\mathbf{E}(\mathbf{x}) e^{-i \omega t}$, Eqs. (1) and (2) become the familiar Helmholtz equations:

$$
\begin{aligned}
& \left(\nabla^{2}+k^{2}\right) \mathbf{H}=0, \\
& \left(\nabla^{2}+k^{2}\right) \mathbf{E}=0 .
\end{aligned}
$$

The wave vector $k$ and index of refraction $\eta$ are defined as

$$
\begin{gathered}
k=\frac{\omega}{c} \sqrt{\varepsilon \mu}, \\
\eta=\sqrt{\frac{\varepsilon}{\mu}} .
\end{gathered}
$$

Solutions to Eqs. (3) and (4) in spherical coordinates traditionally take the form of vector multipole functions (also referred to as vector spherical wave functions). The vector multipole functions are generated from a vector potential $\boldsymbol{\Psi}$ defined as

$$
\boldsymbol{\Psi}=\sum_{n=0}^{\infty} \sum_{m=-n}^{+n} z_{n}(k r) \mathbf{Y}_{n m}^{n}(\theta, \varphi)
$$

The radial function $z_{n}(k r)$ represents either a spherical Bessel function $\left[j_{n}(k r)\right]$ or a spherical Hankel function of the first or second kind $\left[h_{n}^{(1)}(k r)\right.$ or $\left.h_{n}^{(2)}(k r)\right]$. The vector spherical harmonic $\mathbf{Y}_{n m}^{l}(\theta, \varphi)$ is a pure-orbital vector spherical harmonic and is defined with Clebsch-Gordan coefficients, scalar spherical harmonics, and helicity basis vectors as follows: ${ }^{32-34}$

$$
\begin{aligned}
\mathbf{Y}_{n m}^{l}(\theta, \varphi)= & C_{l, m+1,1,-1}^{n, m} Y_{l, m+1} \mathbf{e}_{-1}+C_{l, m, 1,0}^{n, m} Y_{l, m} \mathbf{e}_{0} \\
& +C_{l, m-1,1,1}^{n, m} Y_{l, m-1} \mathbf{e}_{+1} .
\end{aligned}
$$

The vector multipole functions are derived from the vector potential in the following manner: 


$$
\begin{gathered}
\mathbf{V}=\frac{1}{k} \nabla \times \mathbf{\Psi}, \\
\mathbf{W}=\frac{1}{k^{2}} \nabla \times(\nabla \times \mathbf{\Psi}) .
\end{gathered}
$$

The resulting functions are complete and orthogonal multipole expansions for transverse fields:

$$
\begin{gathered}
\mathbf{V}=\sum_{n=0}^{\infty} \sum_{m=-n}^{+n}\left[i \sqrt{\frac{n+1}{2 n+1}} z_{n-1}(k r) \mathbf{Y}_{n m}^{n-1}(\theta, \varphi)\right. \\
\left.-i \sqrt{\frac{n}{2 n+1}} z_{n+1}(k r) \mathbf{Y}_{n m}^{n+1}(\theta, \varphi)\right], \\
\mathbf{W}=\sum_{n=0}^{\infty} \sum_{m=-n}^{+n} z_{n}(k r) \mathbf{Y}_{n m}^{n}(\theta, \varphi) .
\end{gathered}
$$

The $\mathbf{V}$ and $\mathbf{W}$ vector multipoles are similar to the vector spherical wave functions of Stratton, ${ }^{35}$ the vector multipoles of Rose, ${ }^{36}$ Edmonds, ${ }^{37}$ and Greiner and Maruhn, ${ }^{32}$ and the vector spherical harmonic of Jackson. ${ }^{38} \mathbf{V}$ and $\mathbf{W}$ are related to the electric and magnetic fields as follows:

$$
\begin{aligned}
& \mathbf{E}(\mathbf{r})=k[\mathbf{V}(\mathbf{r})+i \mathbf{W}(\mathbf{r})], \\
& \mathbf{H}(\mathbf{r})=k[\mathbf{V}(\mathbf{r})-i \mathbf{W}(\mathbf{r})] .
\end{aligned}
$$

The initial waves incident on the spherical particles in the suspensions are modeled as plane waves. At least three different partial wave expansions have been presented in the literature for transverse vector plane waves, including those by Stratton, ${ }^{35}$ Jackson, ${ }^{38}$ and Greiner and Maruhn. ${ }^{32}$ Grandy further derived expansions for plane-polarized waves from Jackson's circularly polarized waves. ${ }^{39}$ Mathematical and numerical analyses show, however, that only the expansions of Greiner and Maruhn are correct and converge properly. Adapting their expressions, the partial wave expansions for transverse waves propagating in the $z$ direction and polarized in the $x$ and $y$ directions are the following:

$$
\mathbf{e}_{x} e^{i k z}=\sum_{n} i^{n} \sqrt{\pi(2 n+1)}\left[\mathbf{W}_{n,+1}+\mathbf{V}_{n,+1}+\mathbf{W}_{n,-1}-\mathbf{V}_{n,-1}\right]
$$

$\mathbf{e}_{y} e^{i k z}=(-i) \sum_{n} i^{n} \sqrt{\pi(2 n+1)}\left[\mathbf{W}_{n,+1}+\mathbf{V}_{n,+1}-\mathbf{W}_{n,-1}+\mathbf{V}_{n,-1}\right]$

Simulation of multiple scattering in a suspension of spherical particles requires scattering solutions for the individual spheres for an arbitrary incident wave field. This solution is the well-known Mie scattering solution. For scattering from a single sphere, there will be an incoming incident field, a refracted interior field, and an outgoing scattered field for each of the $\mathbf{V}$ and $\mathbf{W}$ wave fields. Each wave field component will also have an associated amplitude coefficient.

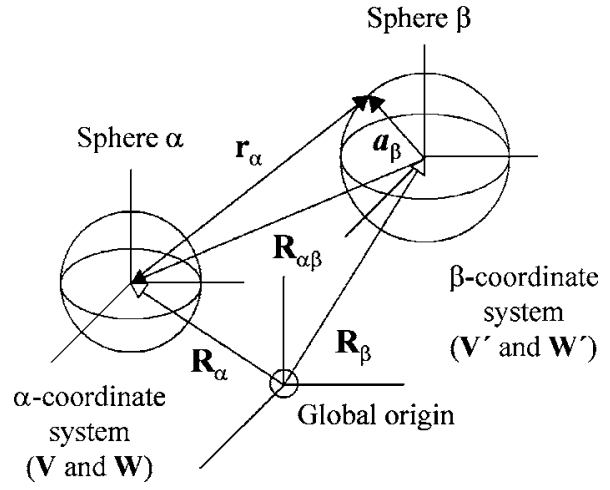

FIG. 1. Position $\left(\mathbf{R}_{\alpha}\right.$ and $\left.\mathbf{R}_{\beta}\right)$ and translation $\left(\mathbf{r}_{\alpha}\right)$ vectors for addition theorems with respect to local and global coordinates for spheres $\alpha$ and $\beta$.

Solution of Mie scattering from dielectric spheres has been published extensively in the literature, so it will not be reproduced here.

The scattered waves from each particle propagate to the other particles in multiple scattering and modify the other particles' scattering interactions. Since the vector multipole functions are specific to each particle's coordinate system, the scattered wave fields from each particle must be transformed into the coordinate systems of the other particles in order to compute the modified interactions. These transformations are the following:

$$
\begin{aligned}
& \mathbf{V}_{\nu \mu}^{\prime}=\sum_{n=0}^{\infty} \sum_{m=-n}^{n}\left(S_{\nu \mu}^{n m} \mathbf{V}_{n m}+T_{\nu \mu}^{n m} \mathbf{W}_{n m}\right), \\
& \mathbf{W}_{\nu \mu}^{\prime}=\sum_{n=0}^{\infty} \sum_{m=-n}^{n}\left(S_{\nu \mu}^{n m} \mathbf{W}_{n m}+T_{\nu \mu}^{n m} \mathbf{V}_{n m}\right),
\end{aligned}
$$

where $\mathbf{V}_{n m}$ and $\mathbf{W}_{n m}$ are wave fields scattered from the originating sphere (designated $\alpha$ ), and are, therefore, in the $\alpha$ sphere's coordinate system $\left(r_{\alpha}, \theta_{\alpha}\right.$, and $\left.\varphi_{\alpha}\right) . \mathbf{V}_{\nu \mu}^{\prime}$ and $\mathbf{W}_{\nu \mu}^{\prime}$ are the same wave fields incident on a second sphere (designated $\beta$ ), and are, therefore, in the $\beta$ sphere's coordinate system $\left(r_{\beta}, \theta_{\beta}\right.$, and $\left.\varphi_{\beta}\right)$. Equations (17) and (18) transform the wave fields with expansions containing the original $(\alpha)$ multipole functions and translation coefficients denoted as $S_{\nu \mu}^{n m}$ and $T_{\nu \mu}^{n m}$. These translation coefficients are also known as the vector addition theorems for spherical wave functions.

Figure 1 shows the geometric relationship for the field translations between the two spheres. The position of sphere $\alpha$ with respect to sphere $\beta$ is $\mathbf{R}_{\alpha \beta}=\mathbf{R}_{\alpha}-\mathbf{R}_{\beta}$, where $\mathbf{R}_{\alpha}$ and $\mathbf{R}_{\beta}$ are the global position vectors for the two spheres. The translation coefficients are only valid on the surface of sphere $\beta\left(r_{\beta}=a_{\beta}\right)$, and the center of sphere $\alpha$ must lie outside of sphere $\beta$. Although addition theorems can also be derived for spheres embedded within larger spheres $\left(a_{\beta}>R_{\alpha \beta}\right)$, this work only considered spheres external to each other and nonoverlapping $\left(a_{\beta}<R_{\alpha \beta}\right)$.

Many methods have been published for deriving the vector addition theorems, including directly transforming the coordinates in the vector multipole functions and applying vari- 
ous identities and relationships to simplify the solution. ${ }^{40}$ Another approach is to expand the vector multipoles in the $\alpha$ coordinate system as a series comprising expansion coefficients and vector spherical harmonics in the $\beta$ coordinate system. ${ }^{41,42}$ The expression can then be integrated in a manner similar to a Fourier series to determine the translation coefficients, which are the following:

$$
\begin{gathered}
S_{\nu \mu}^{n m}=Z_{\nu, \nu, \mu}^{n, n, m}, \\
T_{\nu \mu}^{n m}=-i \sqrt{\frac{2 \nu+1}{\nu+1}} Z_{\nu-1, \nu, \mu}^{n, n, m}=i \sqrt{\frac{2 \nu+1}{\nu}} Z_{\nu+1, \nu, \mu}^{n, n, m}, \\
=i \sqrt{\frac{2 n+1}{n+1}} Z_{\nu, \nu, \mu}^{n-1, n, m}=-i \sqrt{\frac{2 n+1}{n}} Z_{\nu, \nu, \mu}^{n+1, n, m},
\end{gathered}
$$

where

$$
\begin{aligned}
Z_{\lambda, \nu, \mu}^{l, n, m}\left(\mathbf{R}_{\alpha \beta}\right)= & \sum_{p=|l-\lambda|}^{l+\lambda}\left\{i^{\lambda-l-p} h_{p}^{(1)}\left(k R_{\alpha \beta}\right) Y_{p, m-\mu}\left(\Theta_{\alpha \beta}, \Phi_{\alpha \beta}\right)\right. \\
& \times C_{\lambda, 0, p, 0}^{l, 0} \sqrt{\frac{4 \pi(2 \lambda+1)(2 p+1)}{2 l+1}} \\
& \left.\times \sum_{\tau=-1}^{1} C_{l, m-\tau, 1, \tau}^{n, m} C_{\lambda, \mu-\tau, 1, \tau}^{\nu, \mu} C_{\lambda, \mu-\tau, p, m-\mu}^{l, m-\tau}\right\} .
\end{aligned}
$$

Although the vector addition theorems and corresponding translation coefficients can be calculated directly, recurrence formulas significantly reduce the number of operations for their computation. ${ }^{43,44}$

\section{B. Computational approach}

The IM model first calculates the scattered wave fields for each particle in the system due to an initial plane wave. The scattered wave fields are then translated between all particle pairs and summed at each particle. The new incident wave field (initial plane wave+scattered wave fields) is then used to compute revised scattered wave fields from each particle. This process is repeated iteratively until the scattered wave fields converge (i.e., no change in wave field amplitudes between consecutive iterations). Each iteration represents a successive order of scattering, where the first iteration is first-order multiple scattering, the second iteration is secondorder multiple scattering, and so forth. The Cartesian components ( $x, y$, and $z$ amplitudes) of the $\mathbf{V}$ and $\mathbf{W}$ multipole fields are then calculated for an array of grid points in the suspension. These grid-point values can be used to generate either volume-averaged field values or images of the electromagnetic field distribution at the particle level.

Computation limits for the simulations include the maximum multipole expansion order $\left(n_{\max }\right)$ for computing the field and translation coefficient expansions, and the convergence criteria (precision limit) for stopping the iterations. Computer algorithms for the model were written, debugged, and compiled in FORTRAN 90 with double precision. Simulations were performed on a personal desktop computer with 2.0 gigabytes random access memory and a $3.2 \mathrm{GHz}$ processor. Computation times varied from 1 to $72 \mathrm{~h}$.
Calculation of the translation coefficients for all particle pairs in the suspension is computationally intensive and exceeds the dynamic memory capabilities of most desktop computers. Since many particle-pair configurations, and thus, their translation coefficients, are multiply redundant in lattices, a template method was developed that computed the translation coefficients for a single particle surrounded by a lattice. The particle-particle configurations in the suspension were then correlated to the particle-lattice configurations in the template and the corresponding translation coefficients applied to the suspension.

A similar template method was developed for the random microstructures. However, in this case, the lattice surrounding the central particle in the template was replaced with a grid constructed from tessellations of spherical shells surrounding the particle. The grid-point spacing for the random template was additionally much smaller than for the lattice templates and on the order of a particle radius. In the computation process, the randomly configured particles in the suspension are correlated to the nearest grid point in the template. Applied to random suspensions, the template method, therefore, substitutes the exact translation coefficients with approximate coefficients derived from the random template. Correspondingly, a finer template grid provides more accurate translation coefficients. Testing showed that this method provided sufficiently accurate results without compromising the random nature of the microstructure.

Representative suspensions of spherical particles with a monomodal size distribution were generated for sc, bcc, fcc, hexagonal-close-packed (hcp), and random microstructures. Particle-volume fraction varied from 0.025 to 0.60 . The simulated suspensions were configured into spherical, cubic, and cylindrical shapes of fixed volume. Particle-volume fraction was varied by increasing or decreasing the number of particles in the volume. For cylindrical suspensions, the cylinder's diameter was equal to its length and its axis was aligned with the direction of the wave propagation ( $z$ axis). The suspensions contained from 127 to 3587 particles (fcc, 0.025-0.60 volume fraction). One of the random microstructures was constructed using a Monte Carlo type particle dropping routine and by scaling the particle coordinates to create the desired volume fraction. ${ }^{45}$ The other random microstructure was generated from a different but more wellknown Monte Carlo algorithm developed to compare the structures of simple liquids to random close packings. ${ }^{46,47}$

The particles were modeled as glass spheres $(\varepsilon=7.6)$ in air $(\varepsilon=1.0)$. Except for simulations to determine the frequency dependence of the effective permittivity, the diameter of the glass spheres was $1.0 \mathrm{~cm}$ and the simulations were performed at $10 \mathrm{MHz}$. Since the particle and matrix permittivities were kept constant with frequency, the simulations could also apply to $500 \mu \mathrm{m}$ particles (used in the experimental measurements) at $200 \mathrm{MHz}$. Although the electric field arises from a plane wave, the variation in the electric field across the suspension was small since the simulations were performed at very low frequencies.

The effective permittivity was obtained by averaging the E and $\mathbf{D}$ fields over a representative volume containing the particles and matrix. The effective macroscopic dielectric constant was computed from the volume-averaged fields using the definition given by Sihvola: ${ }^{3}$ 


$$
\varepsilon_{e f f}=\frac{\langle\mathbf{D}\rangle}{\langle\mathbf{E}\rangle} .
$$

The averaging was performed by evaluating the $\mathbf{E}$ and $\mathbf{D}$ fields on a uniformly spaced three-dimensional grid containing $31^{3}=29791$ points for the ordered lattices and $37^{3}$ $=50653$ points for the random suspensions. For the ordered lattices, the evaluation grid spanned a unit cell of the lattice located in the center of the suspension. For the random suspensions, the evaluation grid comprised a cube containing from approximately 70 particles $(0.10$ volume fraction) to 420 particles (0.60 volume fraction). Since the electric field was polarized in the $x$ direction in the simulations, only the $x$ components of the electric field and electric displacement were used to compute the effective permittivity. This was equivalent to the use of a parallel-plate capacitor for measuring the dielectric constant, and provided results physically comparable to experimental methods.

\section{Experimental methods}

The time domain reflectometry (TDR) method was used to measure the effective permittivity of samples composed of randomly close-packed spheres. ${ }^{48,49}$ TDR measures the propagation velocity of a step voltage pulse that has a wide bandwidth, usually $10 \mathrm{kHz}-3 \mathrm{GHz}$. The approximate measurement frequency for the TDR technique is dependent on probe design, and was approximately $400 \mathrm{MHz}$ for this work. A Tektronix 1502C TDR cable tester was used for the experiments to measure the effective permittivity. The TDR was connected to a personal computer which was used to collect and analyze wave forms using software developed by Heimovaara and de Water. ${ }^{50}$ The software fits tangent lines to the wave form to locate the end reflection, calculate the travel time of the pulse from the end of the probe, and determine the relative permittivity of the sample. The TDR was connected via a $1.8 \mathrm{~m}, 50 \Omega$ (RG 58) coaxial cable to a coaxial waveguide constructed from steel, $200 \mathrm{~mm}$ long with an internal diameter of $26.5 \mathrm{~mm}$ and a $6 \mathrm{~mm}$ diameter inner electrode. The waveguide was calibrated for effective length using de-ionized water and air.

Spherical glass beads (Mo-Sci Corp., Rolla, MO, USA) made of soda lime silicate glass were chosen as a reference spherical material. These precision $500 \mu \mathrm{m}$ spheres had a particle density of $2.49 \mathrm{~g} \mathrm{~cm}^{-3}$, determined using the excluded volume method. The permittivity of the spheres was measured to be 7.6 using the immersion method. ${ }^{51}$ Measurements were made by filling the coaxial probe with air or a dielectric fluid and making a background permittivity measurement at room temperature. A preweighed sample of beads was then carefully poured into the coaxial cell. For a dielectric fluid background, a portion of the fluid was first removed to account for the displacement produced by the beads. The container was tapped gently to allow the spheres to pack to the desired volume fraction and the effective permittivity was measured again.

\section{Effective-medium approximations}

Three effective-medium approximations were used for comparison to the simulation results and experimental data.
The first of these approximations was the Maxwell Garnett (MG) equation: ${ }^{4}$

$$
\varepsilon_{e f f}=\varepsilon_{0}+3 f \varepsilon_{0}\left[\frac{\varepsilon_{1}-\varepsilon_{0}}{\varepsilon_{1}+2 \varepsilon_{0}-f\left(\varepsilon_{1}-\varepsilon_{0}\right)}\right] .
$$

The particle, matrix, and effective permittivities are $\varepsilon_{1}, \varepsilon_{0}$, and $\varepsilon_{\text {eff }}$, respectively. The particle-volume fraction is $f$. Equation (23) is a first-order approximation accounting only for dipole-dipole interactions between particles in the suspension.

The second approximation is the self-similar or selfconsistent model of Sen et al.: 6

$$
f=1-\left(\frac{\varepsilon_{1}-\varepsilon_{e f f}}{\varepsilon_{1}-\varepsilon_{0}}\right)\left(\frac{\varepsilon_{0}}{\varepsilon_{e f f}}\right)^{1 / 3} .
$$

Note that the effective permittivity $\varepsilon_{e f f}$ is implicit. Equation (24) is also known as the differential effective-medium (DEM) approximation, ${ }^{5}$ which models the suspension as a fractal dispersion of particles. In the DEM model, a shell of matrix material surrounds each particle. Each shell further comprises smaller particles with their own shells of matrix material, and so on. The DEM approximation is, therefore, considered most applicable to random pore networks in geologic media. For dispersions of monomodal-sized spheres, the DEM approximation typically provides an upper limit for the effective permittivity when the inclusion permittivity is higher than the background.

The third approximation is from Sihvola and Kong (SK), and combines the MG equation [Eq. (25)], symmetric effective-medium approximation (SEMA), and coherent potential formula into one equation: $:^{7,52-54}$

$$
f=\left(\frac{\varepsilon_{e f f}-\varepsilon_{0}}{\varepsilon_{1}-\varepsilon_{0}}\right)\left[\frac{\varepsilon_{1}+2 \varepsilon_{0}+3 \sigma\left(\varepsilon_{e f f}-\varepsilon_{0}\right)}{\varepsilon_{e f f}+2 \varepsilon_{0}+3 \sigma\left(\varepsilon_{e f f}-\varepsilon_{0}\right)}\right] .
$$

The parameter $\sigma$ is adjustable and yields the MG formula [Eq. (23)] for $\sigma=0$, the SEMA model for $\sigma=2 / 3$, and the coherent potential formula for $\sigma=1$. Again, the effective permittivity $\varepsilon_{\text {eff }}$ is implicit.

\section{E. Lattice models}

Equations (23)-(25) do not provide for any microstructural input for computing the effective permittivity, and are, therefore, low-order approximations. In contrast, Torquato developed a microstructure-dependent model based on a perturbation expansion of the polarization (here, the polarization is expressed as an integral equation containing Green's function for the electric field). ${ }^{8}$ The expansion yields a set of microstructure-dependent parameters known as point probability functions. Truncation of the expansion provides the following approximate formula for three-dimensional microstructures:

$$
\varepsilon_{e f f}=\varepsilon_{0}\left[\frac{1+2 f \delta-2(1-f) \zeta \delta^{2}}{1-f \delta-2(1-f) \zeta \delta^{2}}\right],
$$

where $\zeta$ is the microstructure-dependent three-point parameter and is a function of $f$. The parameter $\delta$ is 


$$
\delta=\frac{\varepsilon_{1}-\varepsilon_{0}}{\varepsilon_{1}+2 \varepsilon_{0}}
$$

Equation (26) will subsequently be referred to as the Torquato (TQ) model. Torquato provides three-point parameter values for $f=0.10-0.70$ for sc, bcc, fcc, and random microstructures of impenetrable spheres, and for $f$ $=0.10-0.90$ for penetrable spheres ${ }^{8}$

Since the positions of inclusions on a periodic lattice are well defined and can be concisely represented with unit cells and symmetry operations, the effective properties of these microstructures should be computable to very high accuracy using multipole expansion methods. ${ }^{9-12}$ These methods were first derived by Rayleigh, and formulate the electrostatic fields for a sphere in a lattice as follows:

$$
\sum_{n, m} A_{n m} r_{\beta}^{n} Y_{n m}\left(\theta_{\beta}, \varphi_{\beta}\right)=\sum_{i \neq 0} \sum_{n, m} \frac{B_{n m}}{R_{i \beta}^{n+1}} Y_{n m}\left(\Theta_{i \beta}, \Phi_{i \beta}\right)+E_{0} z
$$

Here, $A_{n m}$ represents the field coefficients for the central sphere (sphere $\beta$ ), $B_{n m}$ represents the field coefficients for all of the other spheres in the lattice, and the summation over $i$ is for all the other spheres except sphere $\beta$. The coordinates $R_{i \beta}, \Theta_{i \beta}, \Phi_{i \beta}$ describe the relative position vector between the $i$ th sphere and sphere $\beta$ (equivalent to $\mathbf{R}_{\alpha \beta}$ in Fig. 1), and $E_{0} z$ is the applied external field in the $z$ direction. Equation (28) is known as the Rayleigh identity, and with application of addition theorems and lattice symmetries, provides a linear system of equations for solution of $A_{n m}$ and $B_{n m}$.

Rayleigh originally solved Eq. (28) for the sc lattice. ${ }^{9}$ Rayleigh's solution, however, contained a number of mathematical errors. McPhedran and McKenzie corrected these errors in their solution for the sc lattice. ${ }^{10}$ McKenzie et al. further presented solutions for the bcc and fcc lattices. ${ }^{11}$ These revised solutions, however, incorrectly omitted azimuthal terms $(m \neq 0)$ on the assumption that they were negligible. Sangani and Acrivos further refined the Rayleigh method by including calculations having accuracies to the ninth order and all of the azimuthal terms. ${ }^{55}$ Finally, Cheng and Torquato corrected a sign error in the Sangani-Acrivos solutions. $^{12}$

Cheng and Torquato provided an exact solution for the effective conductivity up to multipole order $n=9$ for sc, bcc, and fcc lattices of inclusions with interfacial resistance. ${ }^{12}$ In terms of effective conductivity and by neglecting the resistance, this solution is

$$
\varepsilon_{e f f}=\varepsilon_{0}\left(1-\frac{3 f}{D}\right)
$$

where

$$
\begin{aligned}
D= & -\tau_{1}^{-1}+f+c_{1} \tau_{3} f^{10 / 3}+c_{2} \tau_{5} f^{14 / 3}+c_{3} \tau_{3}^{2} f^{17 / 3} \\
& +c_{4} \tau_{7} f^{6}+c_{5} \tau_{3} \tau_{5} f^{7}+c_{6} \tau_{9} f^{22 / 3}+O\left(f^{25 / 3}\right),
\end{aligned}
$$

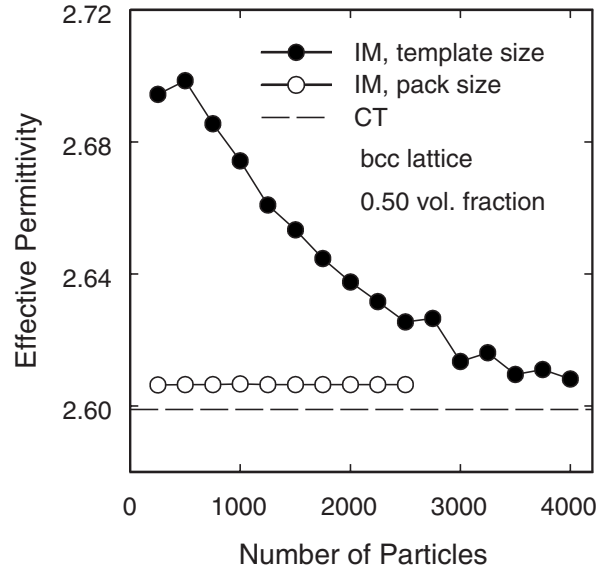

FIG. 2. Effect of template size and particle pack size on iterative multipole (IM) model results for the effective permittivity of $1.0 \mathrm{~cm}$ glass spheres in air. Results are for a bcc lattice and 0.50 particlevolume fraction, and are compared against the results from the Cheng-Torquato (CT) model for an infinite lattice.

$$
\tau_{i}=\frac{i\left(\varepsilon_{1}-\varepsilon_{0}\right)}{i\left(\varepsilon_{1}+\varepsilon_{0}\right)+\varepsilon_{0}} .
$$

The parameters $c_{1}, c_{2}, c_{3}, c_{4}, c_{5}$, and $c_{6}$ are microstructuredependent numerical constants for the sc, bcc, and fcc lattices. Equations (29)-(31) provide standard exact lattice solutions derived from the Rayleigh method and will be subsequently referred to as the Cheng-Torquato (CT) model.

\section{RESULTS}

\section{A. Lattice simulations}

Simulations were first performed on a bcc suspension with 0.50 particle-volume fraction to determine the effects of template size and suspension size (particle number) on the IM model's accuracy. The simulations where performed to $n_{\max }=7$. Figure 2 compares the results of these simulations with the value for an infinite lattice from the CT model. The results show that the IM model overpredicts the effective permittivity with small template sizes, but becomes asymptotically more accurate as the template size-and, therefore, the number of nearest interacting neighbors-increases. These results demonstrate that the differences between IM and CT model predictions are at least partly due to the IM model's capability of simulating only a finite number of particle-particle interactions.

Figure 2 also shows that with a sufficiently large template size (4000 possible interacting neighbors in this case) and with the particle-volume fraction held constant $(0.50)$, the size of the suspension (number of particles) has little effect on the effective permittivity in the range of 250-2500 particles. The fact that the size of the suspension had no effect on the predictions was surprising. The model predictions were also found to be insensitive to the shape of the particle pack or suspension (sphere, cube, or cylinder). All subsequent lattice simulations were performed with a 4000particle template, the largest template possible with the personal computing resources available. 


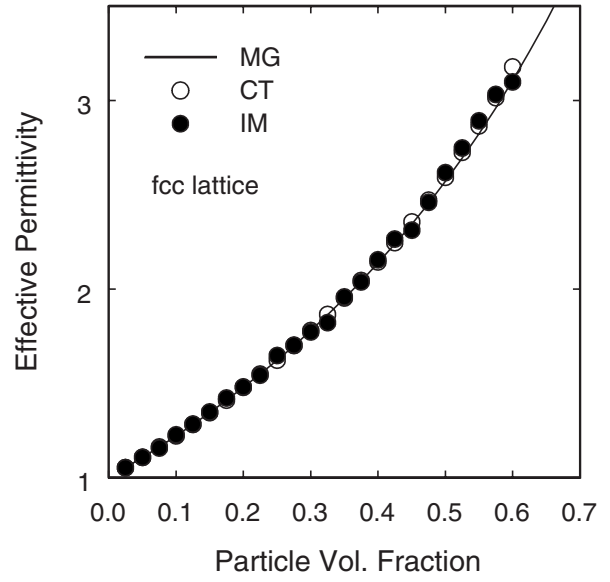

FIG. 3. Effective permittivities for fcc lattices from MG model, CT model, and IM model. Results are for $1.0 \mathrm{~cm}$ glass spheres in air and as a function of particle-volume fraction.

Figure 3 displays the effective permittivities of $1.0 \mathrm{~cm}$ glass spheres in air as a function of particle-volume fraction for the MG model, and for the CT and IM models for fcc lattices. The IM results where computed to $n_{\max }=7$, and the $\mathrm{CT}$ and IM data were modeled with particle-volume fractions in steps of 0.025 . Figure 3 indicates that the models closely agree for dilute suspensions $(\leqslant 0.30$ particle-volume fraction), but that the CT and IM models increasingly deviate from the MG model at higher volume fractions as expected. Additionally, the CT and IM models appear to exhibit good agreement. Similar results were observed for simulations of glass spheres in water where the CT and IM permittivities deviate below the MG predictions due to the background and inclusion permittivity contrast reversal. Differences between the models are difficult to assess, however, when the data are displayed in this format. The results were, therefore, normalized by taking the percent deviation of the effective permittivity from the MG model-a recognized standard model that also forms a lower bound for the predictions when the inclusion permittivity is higher than the background. Figure 4 displays the results of this normalization for the data in Fig. 3. The deviations of the CT and IM models from the MG model are clear, as is the degree of variation in the IM predictions.

Figures 5-8 show normalized effective permittivities for sc, bcc, fcc, and hcp lattices, respectively. For these data sets, the particle-volume fractions were modeled in steps of 0.10 . Figures 5-8 additionally display data for the TQ model and for a single-scattering (SS) model where the iterative steps in the IM computations were disabled. Exact lattice models predicting the permittivity for a hcp structure were not available from the literature. Therefore, TQ and CT fcc models were used for comparison since both hcp and fcc lattices are closepacked structures with local 12-fold coordination. Of the four lattice models, the IM sc model results (Fig. 5) show the closest agreement to the TQ and CT models. The IM bcc and fcc model results (Figs. 6 and 7) display greater scatter. Finally, the IM hcp model produced effective permittivities much greater than predicted by the CT and TQ models for the analogous fcc lattice (Fig. 8).

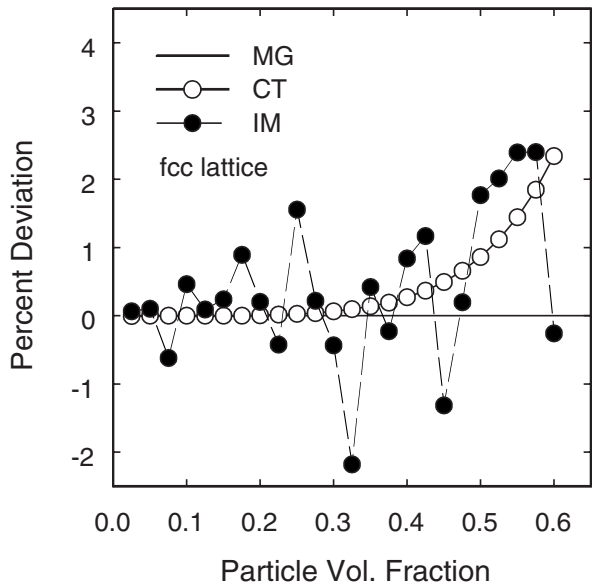

FIG. 4. Effective permittivities for fcc lattices from the CT and IM models. Results are normalized to the MG model by taking the percent deviation, and are for particle-volume fractions in step sizes of 0.025 .

\section{B. Random packing simulations}

Figures 9 and 10 display model results for the two random glass sphere microstructures and experimental data from close-packed glass beads. Comparison with the CT model is not shown since the CT model is only applicable to lattices. The IM predictions for the two microstructures are similar and show very good agreement $(1.76 \%$ for the nonnormalized permittivities) with the TQ model up to 0.50 volume fraction. The IM results underpredict the effective permittivities at higher volume fractions, however, with a $9.72 \%$ deviation from the TQ model at 0.60 . The TQ model also shows good correspondence to the experimental data from random close-packed glass beads. However, the experimental data exhibit a large spread in effective permittivities for the four measurements. (Note that random microstructure 1 did not have a simulated structure with a 0.60 volume fraction due to the nature of the Monte Carlo packing routine and resultant structure formed.)

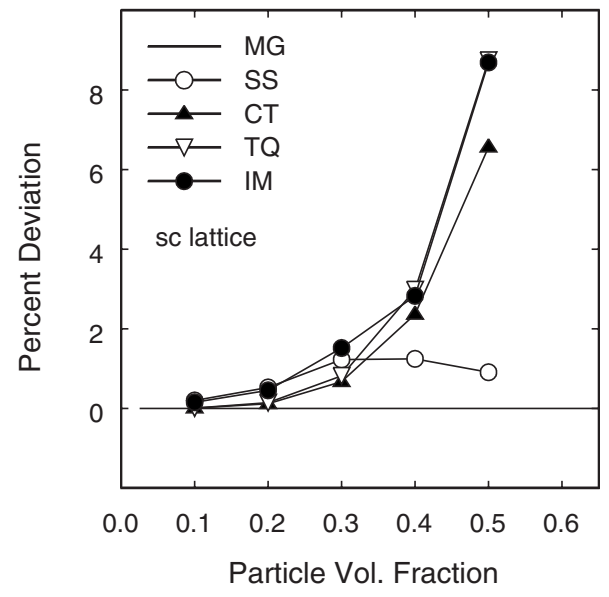

FIG. 5. Effective permittivities for sc lattices from the singlescattering (SS) model, the CT model, the Torquato perturbation expansion (TQ) model, and the IM model. Results are normalized to the MG model, and are for particle volume-fractions in step sizes of 0.10 . 


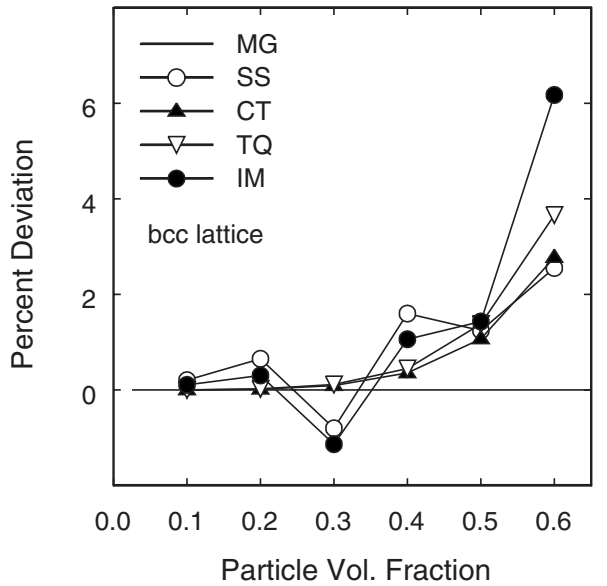

FIG. 6. Effective permittivities for bcc lattices from the SS, CT, TQ, and IM models. Results are normalized to the MG model, and are for particle-volume fractions in step sizes of 0.10 .

One feature of the random microstructure results is that they exhibit higher effective permittivities than the sc, bcc, and fcc lattice results and are more similar to the IM results for the hcp lattice. Figure 11 compares the model results for random microstructure 2 with those from the hcp lattice and two effective-medium approximations. The adjustable parameter $\sigma$ in the SK model was optimized to fit the TQ model $(\sigma=0.14)$. This comparison reveals that the IM results for the random structures most closely resemble the hcp lattice results. The comparison also shows the deficiencies of the effective-medium approximations by either overpredicting the effective permittivities at low volume fractions (DEM model) or underpredicting them at high volume fractions (SK model). Although the trend for the SK model deviates from that of the TQ model at higher volume fractions, the shape of the SK trend follows that predicted by the IM model for the random microstructures and hcp lattice.

\section{Convergence behavior}

The convergence of the multipole simulations is a function of the maximum computed multipole moment $n_{\max }$,

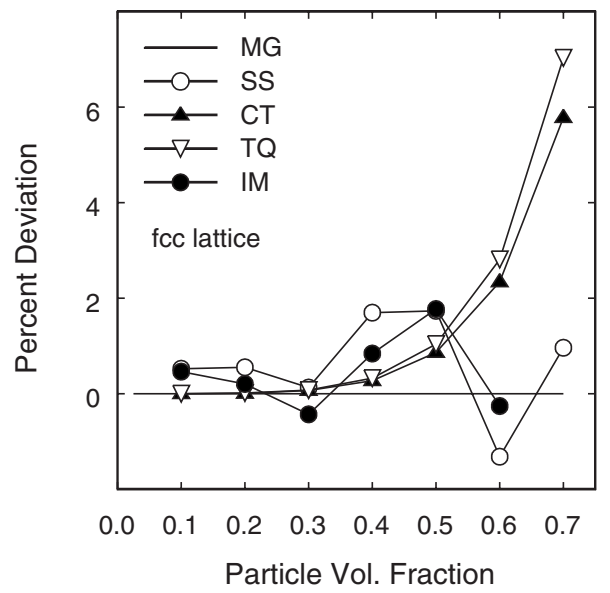

FIG. 7. Effective permittivities for fcc lattices from the SS, CT, TQ, and IM models. Results are normalized to the MG model, and are for particle-volume fractions in step sizes of 0.10 .

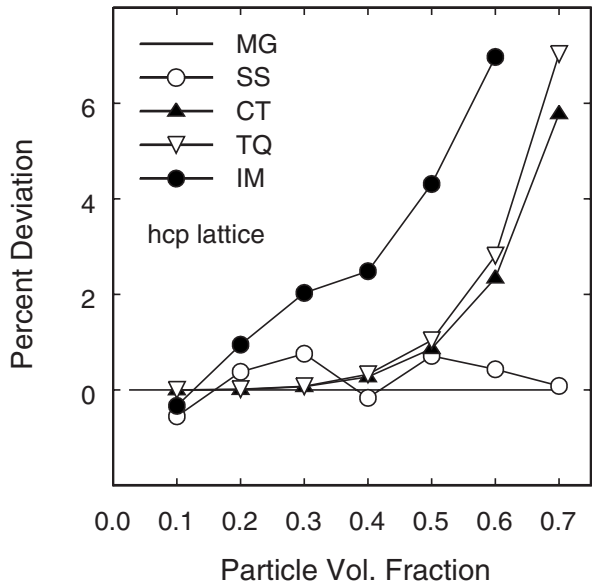

FIG. 8. Effective permittivities for hcp lattices from the SS and IM models, and for fcc lattices from the CT and TQ models. Results are normalized to the MG model, and are for particle-volume fractions in step sizes of 0.10 .

particle-volume fraction, and frequency due to the role of higher-order multipole moments in the convergence of the Mie solutions. These higher-order moments are generated from the partial wave expansions for the initial plane waves and are enhanced by the microstructure. The accuracy of the vector addition theorems is also dependent on $n_{\max }$. The convergence behavior of the vector addition theorems and corresponding translation coefficients has been reported elsewhere and is affected by both particle-particle separation and frequency. ${ }^{42}$

Figure 12 shows the convergence of the effective permittivity values for the multipole simulations as a function of $n_{\max }$ and microstructure. The volume fractions of the lattice and random microstructures were kept constant at 0.50 to provide a valid comparison. Due to the low frequency of the simulations in comparison to the particle diameters and separations, the simulations converge rapidly and plateau by $n_{\max }=7$. Simulations of a bcc suspension at 0.60 volume fraction shows that this plateau extends to $n_{\max }=12$. Due to

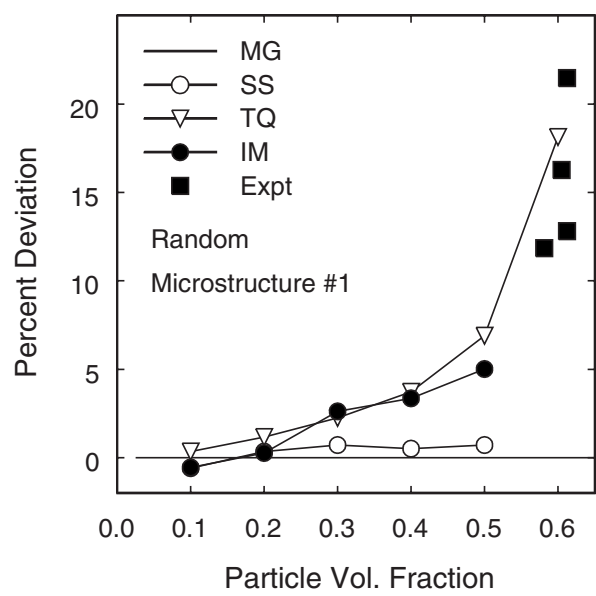

FIG. 9. Effective permittivities for random microstructure 1 from the SS and IM models, random TQ model, and experimental data (Expt) from close-packed glass beads. Results are normalized to the MG model. 


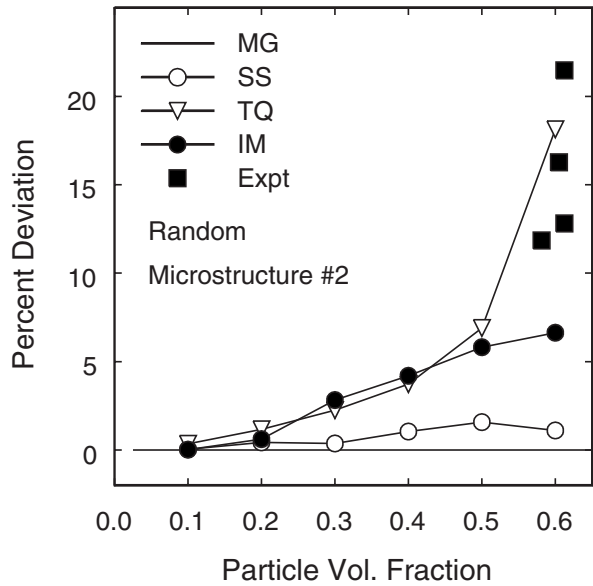

FIG. 10. Effective permittivities for random microstructure 2 from the SS and IM models, random TQ model, and experimental data (Expt) from close-packed glass beads. Results are normalized to the MG model.

the large jump in the permittivity between $n_{\max }=2$ and $n_{\max }$ $=3$ for the sc structure, Fig. 13 omits the sc convergence plot in order to expand and show the other convergence plots in greater detail.

Two features observable in Fig. 13 can be attributed to microstructure. First, the plots for the bcc, fcc, and hcp lattices share a steep step in the permittivity between $n_{\max }=4$ and $n_{\max }=5$. This step probably arises from a common symmetry in these lattices. Second, the plots for the random microstructures are surprisingly smooth in comparison to the lattices. This smoothness can be ascribed to the random microstructures containing a much greater variety of particleparticle orientations than the lattices. These random orientations are averaged over in the computations, resulting in the smooth convergence behavior. A similar smooth convergence curve is observed for random suspensions of glass spheres in water.

Another measure of convergence is the number of iterations required to reach the convergence limit. The conver-

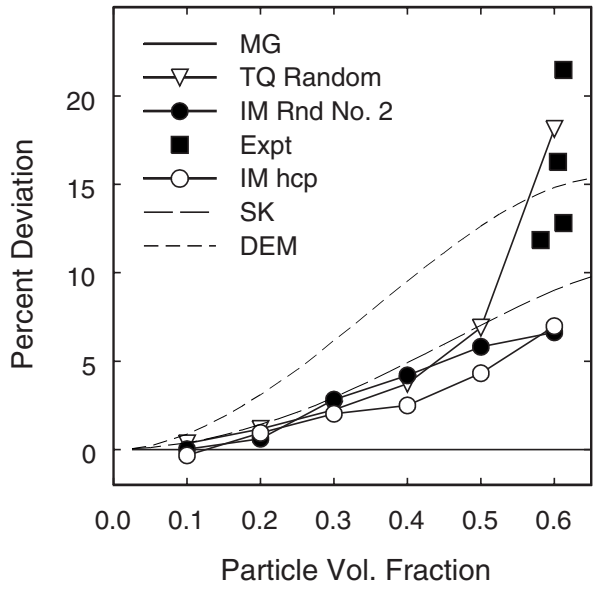

FIG. 11. Effective permittivities for random TQ model, random microstructure 2 (IM model), experimental data (Expt) for closepacked glass beads, hcp structure (IM model), and two mixing formulas (SK and DEM). The SK model was optimized with the adjustable parameter $\sigma$ to fit the TQ model $(\sigma=0.14)$.

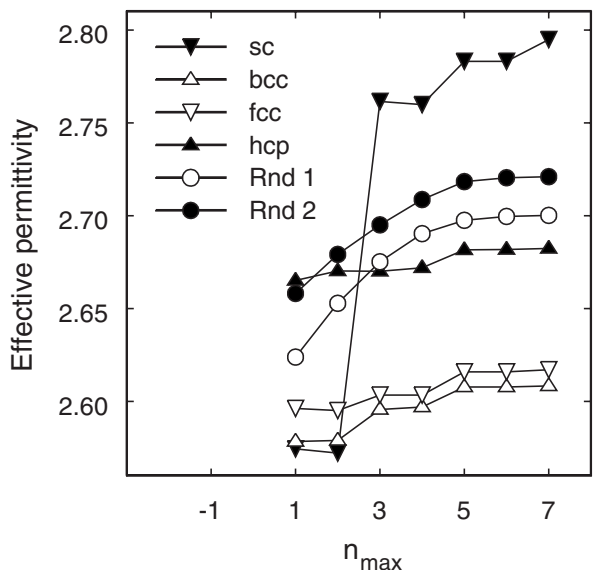

FIG. 12. Convergence of multipole model results as a function of maximum computed multipole order $\left(n_{\max }\right)$ comparing all lattice and random suspensions of glass spheres in air at 0.50 particlevolume fraction.

gence limit is a user-specified lower bound to the fractional change in the computed fields between iteration steps. Once the convergence limit is reached, the iterations terminate. For most of the simulations, this limit was set at $10^{-6}$. Figure 14 displays the number of iterations required to reach the convergence limit for each microstructure and particle-volume fraction. The plots all show a gradually increasing trend in iterations with particle-volume fraction. This trend increases dramatically as the particles approach one another in the microstructures, resulting in nonconvergence at or above 0.60 volume fraction for the bcc, fcc, and hcp simulations and for a $10^{-6}$ limit. Lowering the convergence limit allowed the iterations to converge, but with poorer accuracy. The increase in iterations with volume fraction indicates a rising instability in the iterative solution process. Closer examination of the fractional change in the computed fields of the nonconverging simulations showed that the model partially converges, but then diverges. This partial convergence has

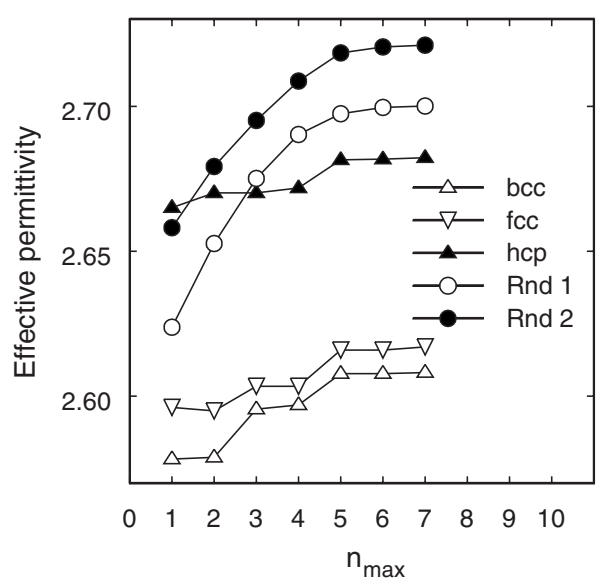

FIG. 13. Convergence of multipole model results as a function of maximum computed multipole order $\left(n_{\max }\right)$ comparing lattice and random suspensions of glass spheres in air at 0.50 particlevolume fraction, excluding sc lattice to accentuate the plots of the other suspensions. 


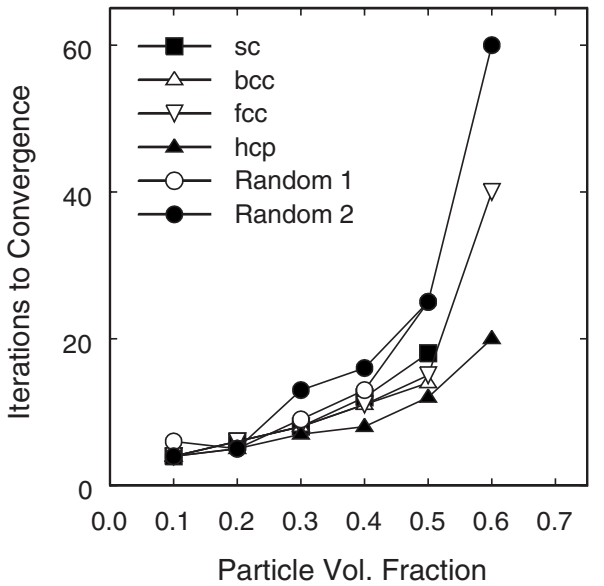

FIG. 14. Number of iterations to convergence (error $<10^{-6}$ ) for multipole model results as a function of particle-volume fraction comparing lattice and random suspensions of glass spheres in air at $n_{\max }=7$.

also been observed in iterative multipole simulations of elastic wave scattering in dispersions of spherical particles. ${ }^{42}$

\section{Frequency dependence}

Simulations were performed for the $0.5-20.0 \mathrm{GHz}$ frequency band for random microstructure 2 and a fcc lattice of $1.0 \mathrm{~mm}$ glass spheres at 0.50 volume fraction to ascertain the frequency dependence (dispersion) of the effective permittivity. Figures 15 and 16 display the results for the fcc lattice and random microstructure 2, respectively. In each figure, data are plotted for three increasingly higher degrees of multiple scattering, starting at single scattering (no nearest neighbors), multiple scattering with 500 nearest neighbors (small template), and multiple scattering with either 4000 (fcc) or 1000 (random) nearest neighbors (large template). The multiple-scattering data terminate before $20 \mathrm{GHz}$ in

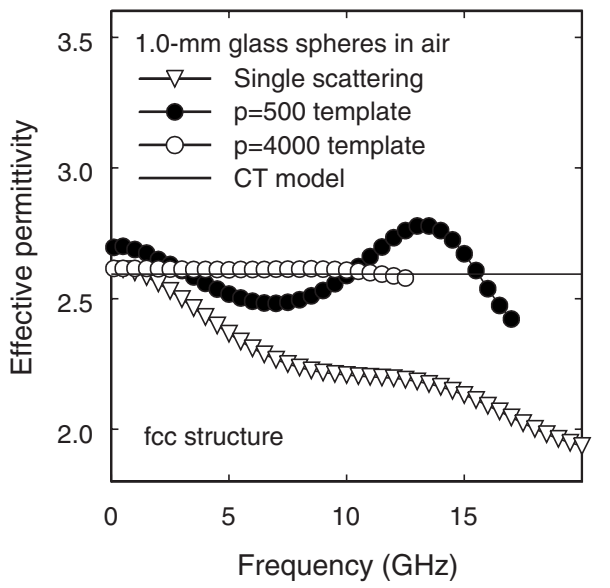

FIG. 15. Computed frequency dependence of the effective permittivity of a fcc suspension of $1.0 \mathrm{~mm}$ glass spheres in air with a particle-volume fraction of 0.50. Results are compared as a function of template size ( $p=$ number of nearest interacting particles) for computing permittivity ( $p=0$ for single scattering).

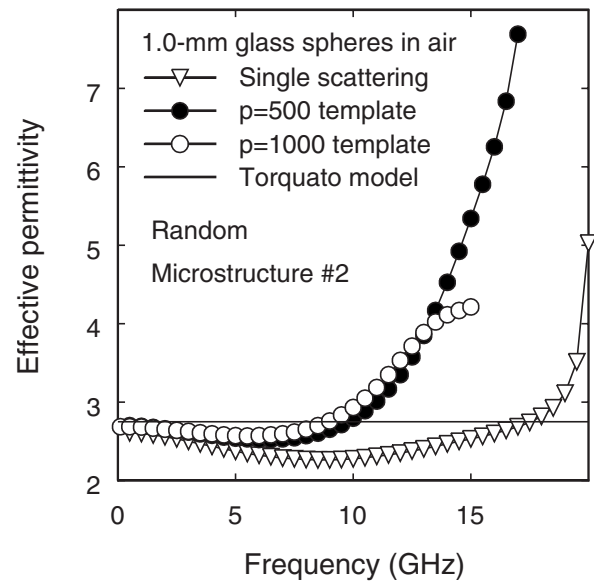

FIG. 16. Computed frequency dependence of the effective permittivity of a random suspension of $1.0 \mathrm{~mm}$ glass spheres in air with a particle-volume fraction of 0.50 . Results are compared as a function of template size ( $p=$ number of nearest interacting particles) for computing permittivity ( $p=0$ for single scattering).

both figures since the number of iterations required for convergence climbed steeply near the end data points and the models became unstable. This is thought to be due to the wavelength of the electromagnetic wave decreasing with increasing frequency and approaching the particle diameter to within an order of magnitude.

The dispersion curves for both the fcc and random microstructures display significant changes as a function of multiple-scattering involvement. They also show striking differences between each other, indicating microstructural effects. The electrostatic values for the fcc lattice (ChengTorquato model) and random microstructure (Torquato model) are also shown for comparison. Of particular note is the steep rise in the effective permittivity for the random microstructure at high frequency $(>10 \mathrm{GHz})$, which is absent for the fcc lattice. Additionally, the fcc dispersion curve flattens dramatically to almost a straight line with increasing template size.

\section{E. Spatial distribution of fields}

One advantage of simulation approaches is their ability to predict the microscopic structure of the electromagnetic fields in space. This is shown in Fig. 17 for the electric field in a random glass sphere/air suspension at 0.50 volume fraction. The dark gray regions in the sphere interiors are due to the suppression of the electric field arising from the permittivity of the glass. The white and light gray regions between particles represent localized areas where the electric field strength is enhanced due to the particle configuration, field superposition, and multiple scattering. Images such as Fig. 17 are useful for diagnosing model performance since they reveal whether the model is producing physically realistic results. They are also valuable in determining anomalously high field concentrations in heterogeneous materials and in identifying regions in the microstructure where electromagnetic fields may be enhanced relative to the external applied field. 


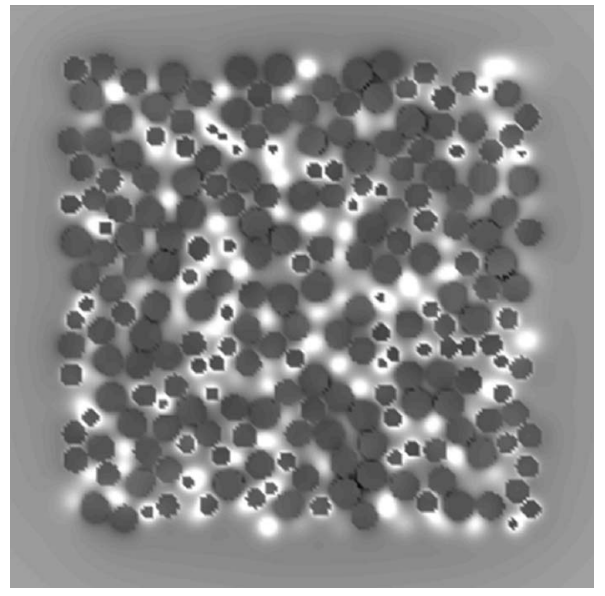

FIG. 17. Model-generated two-dimensional image of the electric field amplitudes in a random three-dimensional suspension of 2870 glass spheres in air with a particle-volume fraction of 0.50. Dark regions represent low field strengths within particles, whereas light regions indicate high field strengths between particles.

\section{DISCUSSION}

\section{A. Lattice results}

When the effective permittivity values from the IM and CT models are compared (sc, bcc, and fcc lattices), the IM model predictions deviate from those of the CT model by less than $3.31 \%$. Figure 2 demonstrates that an important parameter in the IM model with regards to accuracy is the number of nearest interacting neighbors (template size) used in the simulations. With a sufficiently large template and lattice, the asymptotic behavior of the plot in Fig. 2 would eventually bring it into almost perfect agreement with the exact lattice calculations of the CT model.

Although Fig. 3 displays good agreement between the IM and CT models, Fig. 4 shows that the IM model exhibits considerable variation in its predictions when amplified in detail. This variation most likely arises from the changing particle configurations (particle-to-particle orientations and distances) as the particle pack density increases. The IM model variations are also observed in Figs. 6-8 and are the primary source of error in the effective permittivity predictions. The same variation trends in the SS model indicate that the source of the variation is not in the iterative process but more likely in the change in particle configuration. Also, Fig. 2 shows that the variation does not arise solely from a change in particle number (since the particle density was held constant in Fig. 2). Rather, it appears that when the particle density changes, as in Figs. 4-8, the particle-toparticle geometries (angles and distances) change, thereby producing variations in the predicted effective permittivities.

The SS model typically predicted effective permittivity values lower than those of the IM model but higher than those of the MG model (Figs. 5-8). For the random microstructures, the SS model results were most consistent with the MG model (Figs. 9 and 10). The SS model excludes all particle-particle interactions by computing the scattered fields from the particles independently and superimposing the fields without calculation of a mean or average field. The
MG model, on the other hand, statistically averages the particle-particle interactions by computing the local electric field inside a theoretical cavity excised from an effective medium. Since the effective medium is characterized as having an average polarizability, the MG model should account for some particle-particle interactions (specifically dipoledipole interactions) and should, therefore, be more in agreement with the exact lattice (CT model) or perturbation expansion (TQ model) results than the single-scattering (SS model) results. The conclusion of these simulations shows that this appears not to be the case and that the MG model is most consistent with a zero-order approximation that does not account for particle-particle interactions. An explanation that accounts for this conclusion is that multipole moments higher than dipole moments play a much more significant role in the particle-particle interactions than previously believed.

\section{B. Random packing results}

The random microstructure IM models (Figs. 9-11) showed much less scatter in their effective permittivity predictions than the lattice models, particularly in comparison to the bcc and fcc lattices (Figs. 4, 6, and 7). This is due to the fact that random microstructures provide a smoother distribution of particle-to-particle configurations than finite lattices, which are restricted to discrete angles and distances due to the lattice symmetries. This distribution will only be quasicontinuous since the random microstructures are finite. However, the randomization of the particle configurations will minimize large variations in the simulation results as the particle density is changed.

The IM model predictions for the random microstructures are in good agreement with the TQ model up to 0.50 particlevolume fraction, but predict lower values than expected for higher particle densities (Fig. 10). This underprediction may be due to an artifact created by the template method used for the random microstructures. This template limits the nearestneighbor particle positions to grid points on spherical shells, and therefore cannot adequately model particle pairs very close to one another or in contact. The interactions of these close particle pairs will be more significant in random microstructures than in lattices (since some will be present at all volume fractions in the random packings) and will also dominate at high volume fractions.

Surprisingly, the random microstructure results most closely match those of the hcp lattice (Fig. 11), suggesting that the dielectric behavior of the hcp lattice is more similar to that of a random microstructure than a cubic lattice. Additionally, the convergence behavior of the hcp lattice (Fig. 12) has a smoother trend than exhibited by the bcc and fcc lattices due to the lack of a step between $n_{\max }=2$ and $n_{\max }$ $=3$. Therefore, the hcp convergence behavior appears to more closely match that of the random microstructures. The similarities between the hcp and random microstructure may be attributable to the lower symmetry of the hcp lattice as compared to the cubic lattices.

The experimental data (Figs. 9-11) displayed considerable spread, possibly since the close packing of glass beads 
in gravity may lead to microstructures with varying degrees of anisotropy or order. Of the two effective medium models examined (SK and DEM), the DEM model produced results that agreed the least with the other modeling approaches. Because of its adjustable variable $\sigma$, the SK model can be optimized to fit any of the other models, and the shape of its trend appears to agree with the IM trends for the random and hcp structures (Fig. 11). However, the SK model predictions deviate from the TQ model approach (Fig. 11) at high particle-volume fractions when optimized across the entire particle density range. Additionally, the SK and IM models fail to predict the experimental data from the close-packed glass spheres. These results illustrate the challenges of modeling suspensions with high $(>0.50)$ particle densities.

\section{Convergence behavior}

The convergence of the multipole models is well behaved for most volume fractions and microstructures. The effective permittivity exhibits a fairly stable convergence by $n_{\max }=7$ for all of the microstructures simulated at 0.50 volume fraction. Similarly, the iterative convergence is well behaved except at high volume fractions $(>0.60)$, where the number of iterations required for convergence increases dramatically. The convergence is also a function of frequency, however, and becomes problematic for frequencies with wavelengths smaller than 20 times the particle diameter.

The convergence behaviors for the bcc, fcc, and hcp lattices as a function of $n_{\max }$ (Fig. 13) are similar with regular steps in the plots. The random suspensions also displayed convergence behavior similar to the bcc, fcc, and hcp lattices, but with the steplike features smoothed out (Figs. 12 and 13). In contrast, the convergence behavior of the sc suspensions differed most significantly from the other microstructures with an anomalously large increase in permittivity from $n_{\max }=2$ to $n_{\max }=3$, indicating that the interactions in the sc lattice may have unusual properties that are not common to the other microstructures. These properties may be related to the coordination number of nearest neighbors in the microstructures since the sc lattice has the lowest coordination number (6) compared to the bcc (8), fcc (12), and hcp (12) lattices. Although the particles in a random suspension will exhibit a range of coordination numbers, hardsphere computations have rigorously shown that the average coordination number is exactly 6 for maximally random jammed packings (0.64-0.65 volume fraction).$^{56}$

Since the simulated random suspensions in this work had no touching particles (i.e., they were not close packed), the number of nearest neighbors for the 0.50 volume fraction suspension (random microstructure 1) was calculated based on the nearest-neighbor distances for the bcc, fcc, and hcp lattices at the same volume fractions. These calculations yielded 7.05 nearest neighbors using the bcc lattice distance, and 7.43 nearest neighbors using the fcc and hcp lattice distances. The number of nearest neighbors for the random suspensions is, therefore, greater than the sc lattice and closer to the bec lattice.

\section{Frequency effects}

The most striking effect of microstructure on effective permittivity has been observed at high frequencies between the fcc and random suspensions (Figs. 15 and 16). Above $10 \mathrm{GHz}$, the effective permittivity for the random suspension sharply increases, whereas for the fcc suspension the effective permittivity slightly decreases. It is currently not known what is causing this effect, although the sharp permittivity increase for the random microstructure may be due to Mie resonances. These resonances would be weakened and broadened by multiple scattering, possibly corresponding to the shift in the shape and position of the dispersion curves in Fig. 16 as a function of template size. Since the random microstructure is truly arbitrary, there should be no other structural effects to influence the Mie resonances such as order or periodicity.

In contrast, the fcc suspension has an ordered structure that would most likely create interference effects that may counter the Mie resonances. These interference effects are also known as photonic band gaps, and it is possible that the $20 \mathrm{GHz}$ region represents the edge of a band gap in the fcc lattice. In contrast to the Mie resonances in the random suspension, multiple scattering should sharpen and intensify the band gap, as possibly indicated by the change in slope of the dispersion curve from single scattering to the 500-particle template (Fig. 15). These photonic band gaps would be absent in the random suspension due to the lack of periodicity. Other modeling parameters that may give rise to spectral features are the finite size of the simulated suspension and particle configuration effects discussed in Sec. IV A. The results from these initial studies indicate, however, that dispersion effects arising from complex microstructures can be modeled, identified, and studied with the presented modeling approach. They also reveal the effects of multiple scattering on effective permittivities at high frequency and the importance of accurately accounting for multiple scattering in electromagnetic computations.

\section{E. Future improvements}

In addition to the current insights provided on the dielectric properties of arbitrary suspensions, the iterative multipole model is capable of modeling more complex microstructures. These structures could include particles of varying sizes and properties, layered particles, hierarchical, nested, or fractal microstructures, and anisotropic microstructures. Modeling these types of microstructures may offer a more complete understanding of the structural and compositional parameters affecting the effective permittivity. Additional improvements could also include the modeling of particles with multiple, noncentrally located inclusions or pores, ${ }^{57}$ and the modeling of nonspherical particles using prolate and oblate spheroidal wave functions. ${ }^{58}$

The computational simplicity of a direct iterative approach also allows larger particle suspensions to be modeled in contrast to other approaches. In the present work, up to 3467 particles were modeled for random suspensions on a desktop computer. This is in comparison to research reported for the $T$-matrix method, which modeled up to only 120 particles ${ }^{24}$ and research using a matrix approach, a central cell containing 200 particles, and modified interactions to account for surrounding periodic cells. ${ }^{31}$ The IM method is 
well suited for parallel computation processes, and implementation of the method on a supercomputing cluster would greatly enhance its capabilities. The convergence limitations of the iterative approach, however, presently prevent its use for simulations at high frequencies and tightly packed suspensions. Work on this problem is essential for future progress on the IM modeling approach.

\section{CONCLUSIONS}

Iterative multipole simulations have been performed for both lattice (sc, bcc, fcc, and hcp) and random suspensions of glass spheres in air and water to calculate their effective permittivities. The suspensions contained approximately $100-3600$ spheres at volume fractions of $0.025-0.60$. The results show fair to very good agreement with both a perturbation expansion model by Torquato ${ }^{8}$ and an exact lattice model using the Rayleigh method by Cheng and Torquato. ${ }^{12}$ The effective permittivities predicted by the iterative multipole model deviated from those predicted by the exact lattice model by less than $3.31 \%$. These deviations varied with particle packing density and can be attributed to effects arising from changes in the particle-to-particle lattice geometries (distances and angular orientations) that correspond to the particle packing density changes. These geometric effects are randomized out in the random microstructure simulations, which show much less variation with particle packing density. No geometric effects were found due to the overall shape of the particle suspensions, however, even in the lattice suspensions. Also, the effective permittivity was observed to remain constant when the particle packing density was kept constant, although the number of particles in the suspension and the resultant size of the suspension were varied.

Comparison of the random microstructure results to those of the lattices indicates that the hcp simulations most closely approximate those of the random microstructures. This is probably due to the lower symmetry of the hcp lattice. The random microstructures also display smoother convergence curves as a function of $n_{\max }$ than the lattices, a result of the random distribution of particle-to-particle orientations in the suspensions. The sc lattice displayed an anomalously large increase in its convergence curve from $n_{\max }=2$ to $n_{\max }=3$. This anomaly can be ascribed to the low coordination number of the sc lattice and possibly to differences due to the symmetry group. It was additionally discovered that the Maxwell Garnett model best approximates a singlescattering model with no multiple-scattering interactions. ${ }^{4}$ Of the effective-medium models tested, the Sihvola-Kong model most closely agreed with the Torquato and iterative multipole models. ${ }^{7,8}$ Comparison of the simulation results with experimental data from randomly close-packed glass beads shows that the iterative multipole model underpredicts the data, indicating a possible deficiency with the model for random microstructures at high particle densities.

The simulations demonstrate that the effective permittivities of spherical particle suspensions will be observably affected by microstructure, particularly at higher particlevolume fractions and higher frequencies. These effects appear to be related to one or more parameters including disorder, lattice symmetries, and coordination number of nearest neighbors. Frequency spectra also reveal that accurate modeling of multiple scattering is essential for predicting the dispersion behavior of the effective permittivity. The present capabilities of the iterative multipole approach additionally allow it to model spatially dependent phenomena and image the microscopic fields in arbitrary suspensions of spherical particles. However, convergence and stability issues limit its current use to low frequencies and long wavelength $(\lambda<20$ times the particle diameter) and to suspensions that are not tightly packed ( $\leqslant 0.60$ particle density).

\section{ACKNOWLEDGMENTS}

The authors wish to thank David G. Tarboton and W. John Raitt for their support in establishing this collaborative effort, I. Lee Davis for generating the random particle microstructures, and David Peak for helpful comments. This work was supported by Utah State University under a Water Initiative Research Grant.
${ }^{1}$ M. Faraday, Experimental Researches in Chemistry and Physics (Taylor and Francis, London, 1991), p. 391.

${ }^{2}$ J. C. Maxwell, A Treatise on Electricity and Magnetism (Clarendon, London, 1873), Vol. 1, p. 435.

${ }^{3}$ A. Sihvola, Electromagnetic Mixing Formulas and Applications (The Institution of Electrical Engineers, London, 1999).

${ }^{4}$ J. C. Maxwell Garnett, Philos. Trans. R. Soc. London, Ser. A 203, 385 (1904).

${ }^{5}$ S. Torquato, Random Heterogeneous Materials: Microstructure and Macroscopic Properties (Springer-Verlag, New York, 2002), p. 459.

${ }^{6}$ P. N. Sen, C. Scala, and M. H. Cohen, Geophysics 46, 781 (1981).

${ }^{7}$ A. Sihvola and J. A. Kong, IEEE Trans. Geosci. Remote Sens. GE-26, 420 (1988).
${ }^{8}$ S. Torquato, J. Appl. Phys. 58, 3790 (1985).

${ }^{9}$ R. S. Rayleigh, Philos. Mag. 34, 481 (1892).

${ }^{10}$ R. C. McPhedran and D. R. McKenzie, Proc. R. Soc. London, Ser. A 359, 45 (1978).

${ }^{11}$ D. R. McKenzie, R. C. McPhedran, and G. H. Derrick, Proc. R. Soc. London, Ser. A 362, 211 (1978).

${ }^{12}$ H. Cheng, Proc. R. Soc. London, Ser. A 453, 145 (1997).

${ }^{13}$ J. Lam, J. Appl. Phys. 68, 392 (1990).

${ }^{14}$ C. Simovski and S. He, J. Appl. Phys. 86, 3773 (1999).

${ }^{15}$ S.-Y. Lu, J. Appl. Phys. 85, 264 (1999).

${ }^{16}$ I. L. Davis, J. Appl. Phys. 67, 955 (1990).

${ }^{17}$ A.-K. Hamid, I. R. Ciric, and M. Hamid, IEE Proc., Part H: Microwaves, Antennas Propag. 138, 565 (1991).

${ }^{18}$ A.-K. Hamid, I. R. Ciric, and M. Hamid, Can. J. Phys. 68, 1157 (1990). 
${ }^{19}$ A.-K. Hamid, I. R. Ciric, and M. Hamid, Can. J. Phys. 68, 1419 (1990).

${ }^{20}$ K. A. Fuller and G. W. Kattawar, Opt. Lett. 13, 1063 (1988).

${ }^{21}$ D. W. Mackowski, Proc. R. Soc. London, Ser. A 433, 599 (1991).

${ }^{22}$ P. C. Waterman, Phys. Rev. D 3, 825 (1971).

${ }^{23}$ B. Peterson and S. Ström, Phys. Rev. D 8, 3661 (1973).

${ }^{24}$ P. R. Siqueira and K. Sarabandi, IEEE Trans. Antennas Propag. 48, 317 (2000).

${ }^{25}$ M. I. Mishchenko, L. D. Travis, and A. Macke, in Light Scattering by Nonspherical Particles, edited by M. I. Mishchenko (Academic, San Diego, 2000), Chap. 6, p. 147.

${ }^{26}$ M. I. Mishchenko, G. Videen, V. A. Babenko, N. G. Khlebtsov, and T. Wriedt, J. Quant. Spectrosc. Radiat. Transf. 88, 357 (2004).

${ }^{27}$ L. Greengard and V. Rokhlin, J. Comput. Phys. 73, 325 (1987).

${ }^{28}$ V. Rokhlin, J. Comput. Phys. 86, 414 (1990).

${ }^{29}$ R. Coifman, V. Rokhlin, and S. Wnadzura, IEEE Antennas Propag. Mag. 35, 7 (1993).

${ }^{30}$ L. Greengard, Science 265, 909 (1994).

${ }^{31}$ K. Hinsen and B. U. Felderhof, Phys. Rev. B 46, 12955 (2000).

${ }^{32}$ W. Greiner and J. A. Maruhn, Nuclear Models (Springer-Verlag, Berlin, 1996), p. 77.

${ }^{33}$ K. S. Thorne, Rev. Mod. Phys. 52, 299 (1980).

${ }^{34}$ D. A. Varshalovich, A. N. Moskalev, and V. K. Khersonskii, Quantum Theory of Angular Momentum (World Scientific, Singapore, 1988).

${ }^{35}$ J. A. Stratton, Electromagnetic Theory (McGraw-Hill, New York, 1941), Chap. 7.

${ }^{36}$ M. E. Rose, Elementary Theory of Angular Momentum (Wiley, New York, 1957), p. 131.

${ }^{37}$ A. R. Edmonds, Angular Momentum in Quantum Mechanics (Princeton University Press, Princeton, 1957), p. 81.

${ }^{38}$ J. D. Jackson, Classical Electrodynamics, 2nd ed. (Wiley, New York, 1975), p. 767.

${ }^{39}$ W. T. Grandy, Jr., Scattering of Waves from Large Spheres (Cam- bridge University Press, Cambridge, 2000), p. 67.

${ }^{40}$ O. R. Cruzan, Q. Appl. Math. 20, 33 (1962).

${ }^{41}$ T. E. Doyle, Ph.D. thesis, Utah State University, 2004.

${ }^{42}$ T. E. Doyle, J. Acoust. Soc. Am. 119, 2599 (2006).

${ }^{43}$ W. C. Chew and Y. M. Wang, J. Electromagn. Waves Appl. 7, 651 (1993).

${ }^{44}$ D. W. Mackowski, J. Opt. Soc. Am. A 11, 2851 (1994).

${ }^{45}$ I. L. Davis and R. G. Carter, J. Appl. Phys. 67, 1022 (1990).

${ }^{46}$ J. L. Finney, Proc. R. Soc. London, Ser. A 319, 479 (1970).

${ }^{47}$ J. L. Finney, Proc. R. Soc. London, Ser. A 319, 495 (1970).

${ }^{48}$ G. C. Topp, J. L. Davies, and A. P. Annan, Water Resour. Res. 16, 574 (1980).

${ }^{49}$ D. A. Robinson, M. Schaap, S. B. Jones, S. P. Friedman, and C. M. K. Gardner, Soil Sci. Soc. Am. J. 67, 62 (2003).

${ }^{50}$ T. J. Heimovaara and E. de Water, Laboratory of Physical Geography and Soil Science Report No. 41, University of Amsterdam, 1993.

${ }^{51}$ D. A. Robinson and S. P. Friedman, J. Geophys. Res. 108, 2076 (2003).

${ }^{52}$ D. A. G. Bruggeman, Ann. Phys. 24, 636 (1935).

${ }^{53}$ D. Polder and J. H. V. van Santen, Physica (Amsterdam) 12, 257 (1946).

${ }^{54}$ L. Tsang, J. A. Kong, and R. T. Shin, Theory of Microwave Remote Sensing (Wiley, New York, 1985).

${ }^{55}$ A. S. Sangani and A. Acrivos, Proc. R. Soc. London, Ser. A 386, 263 (1983).

${ }^{56}$ A. Donev, S. Torquato, and F. H. Stillinger, Phys. Rev. E 71, 011105 (2005).

${ }^{57}$ K. A. Fuller and D. W. Mackowski, in Light Scattering by Nonspherical Particles, edited by M. I. Mishchenko (Academic, San Diego, 2000), Chap. 8, p. 226.

${ }^{58}$ I. R. Ciric and F. R. Cooray, in Light Scattering by Nonspherical Particles, edited by M. I. Mishchenko (Academic, San Diego, 2000), Chap. 4, p. 90. 